\title{
Diagnostic System of Drill Condition in Laminated Chipboard Drilling Process
}

\author{
Bartosz Swiderski ${ }^{1}$, Jaroslaw Kurek $^{1, *}$, Stanislaw Osowski $^{2,3}$, Michal Kruk ${ }^{1}$, Albina Jegorowa ${ }^{1}$ \\ ${ }^{1}$ University of Life Sciences, Warsaw, Poland \\ ${ }^{2}$ Warsaw University of Technology, Warsaw, Poland \\ ${ }^{3}$ Military University of Technology, Warsaw, Poland
}

\begin{abstract}
The paper presents an on-line automatic system for recognition of the drill condition in a laminated chipboard drilling process. Two states of the drill are considered: the sharp enough (still able to drill holes acceptable for processing quality) and worn out (excessive drill wear, not satisfactory from the quality point of view of the process). The automatic system requires defining the diagnostic features, which are used as the input attributes to the classifier. The features have been generated from 5 registered signals: feed force, cutting torque, noise, vibration and acoustic emission. The statistical parameters defined on the basis of the auto regression model of these signals have been used as the diagnostic features. The sequential step-wise feature selection is applied for choosing the most discriminative set of features. The final step of recognition is done by support vector machine classifier working in leave one out mode. The results of numerical experiments have confirmed good quality of the proposed diagnostic system.
\end{abstract}

\section{Introduction}

Use Tool Condition Monitoring (TCM) is an important automatic system to improve the quality and productivity of machine tools without interrupting normal operations $[1,2,3]$. TCM is aiming in detecting or predicting the tool failure. It is composed of sensors, which monitor the signals coming from force, torque, vibration, temperature, acoustic emission, electric current, etc. Many sensors are used for monitoring machining processes and yield an extended set of sensitive features. The registered sensor signals are analysed, transformed and selected by the system to yield the most sensitive features to the monitoring purposes [4,5]. On this basis the decision system is built up to generate automatically the control parameters for the production process. The information from the learning procedure is stored in the system and is used to classify the newly obtained exploitation data. The newly acquired data may be used in retraining the system. With this increasing experience, the system will become more and more reliable in monitoring/control the production.

The paper proposes new solution of TCM in wood industry, directed to monitoring the condition of the drill. Drill wear results in decreasing the cutting edge properties and worsening the product quality [6,7]. The blunting of drill is caused by the mechanical, thermal and chemical influence of the work piece. This is especially important, when the wood based materials with large amount of glue are drilled [6].
Different types of sensors are used in monitoring the sharpness of the drills. The most often applied are the sensors reacting on force, electrical power, acoustic emission, vibration and acoustic pressure $[4,8,9]$. The signals of the mentioned above sensors are more or less correlated with the condition of the tool. However, they are also susceptible to the random interferences and background noise. Many types of sensors are used in a parallel to reduce the influence of the noise. However, the force or torque sensors are relatively complex and difficult in mounting to the cutting tool. On the other side the vibration sensors and current or power sensors are easy to install, but less accurate. The sound signal is similar to the vibration. The acquisition of the sound, usually in the range of frequencies from $20 \mathrm{kHz}$ to $80 \mathrm{kHz}$, can be easily done applying the microphone. However, the acoustic pressure sensors (microphones), are even more sensitive to the background noise.

On the basis of the registered sensor signals the diagnostic features are created and assessed. They will serve as the input attributes to the final classification system. In some publications the diagnostic features are defined directly on the basis of sensor signals in time domain or after their transformation using either Fourier or wavelet transformation [6,10]. Different statistical measures, such as mean value, root mean squared value, power, energy, skewness, kurtosis, etc., are applied. The better correlation of these parameters with the degree of sharpness of the cutting edge of drill the better quality of the features, applied as the input signals to the neural

\footnotetext{
*Corresponding author: jaroslaw_kurek@sggw.pl
} 
classifiers responsible for the recognition of the tool condition $[11,12,13]$.

In this work we apply the diagnostic features defined on the basis of the autocorrelation principle. Five sensor signals, registered in a laminated chipboard drilling process, are exploited. They include feed force $(\mathrm{F})$, cutting torque $(\mathrm{M})$, noise $(\mathrm{C})$, vibration $(\mathrm{V})$ and acoustic emission (A).

The features generated on the basis of these signals should differentiate two classes of drill state: the sharp drill (class 1) and blunt drill (class 2). The first class refers to the tool which is able to drill holes of proper parameters, acceptable from the processing quality point of view. The second class means the drill condition resulting in a not satisfactory quality of the final product.

The paper will present the process of building an automatic diagnostic system for assessing the sharpness of the drill on the basis of the above registered signals. The procedure begins from the acquisition of the sensor signals, then describes the generation of diagnostic features, selection of the best set of these features and final recognition of the condition of drill (sharp or blunt). The support vector machine (SVM) is used as the pattern recognition and classification tool. We used SVM, since it belongs to the most efficient classifiers of good generalization ability.

\section{Data base in experiments}

The data used in experiments have been acquired using Buselatto JET $100 \mathrm{CNC}$ vertical machining centre with a maximum spindle speed of $4500 \mathrm{rpm}$. The data acquisition were done on the laminated chipboard of the dimension $150 \times 35 \times 18 \mathrm{~mm}$ using Faba HW drills of the $12 \mathrm{~mm}$ diameter. The movement of the working unit was caused by a pneumatic actuator with the feed speed equals $1.35 \mathrm{~m} / \mathrm{min}$. The following sensor devices have been applied in experiments: A - acoustic emission measuring system (contact sensor Kistler 8152B, amplifier Kistler 5125B), V - mechanical vibration measuring system (accelerometer Kistler 8141A, amplifier Kistler 5127B), C - noise (sound pressure) measuring system (microphone and preamplifier B\&K 4189, amplifier B\&K NEXUS 2690), F and M dynamometer with Kistler 9345A sensor and ICAM5073A amplifier. The samples have been registered by using two data acquisition cards (NI PCI6111 and NI 6034E) through connection boxes (NI BNC-2110), as shown in Fig. 1. Two acquisition cards of different sampling frequencies: $2 \mathrm{MHz}$ for acoustic emission signal and $50 \mathrm{kHz}$ for vibration and noise signal, respectively, have been applied using the LabVIEW 7 National Instruments application. The measurement system was fully automatic and all measurements have been done on-line in the production process of laminated chipboard drilling.

The acquired data were associated with two conditions of drills. The sharp state (class 1) refers to the tool which is able to drill holes, acceptable from the processing quality point of view. The second state of drill, called blunt, reflect the drill resulting in a processing quality regarded as not satisfactory.

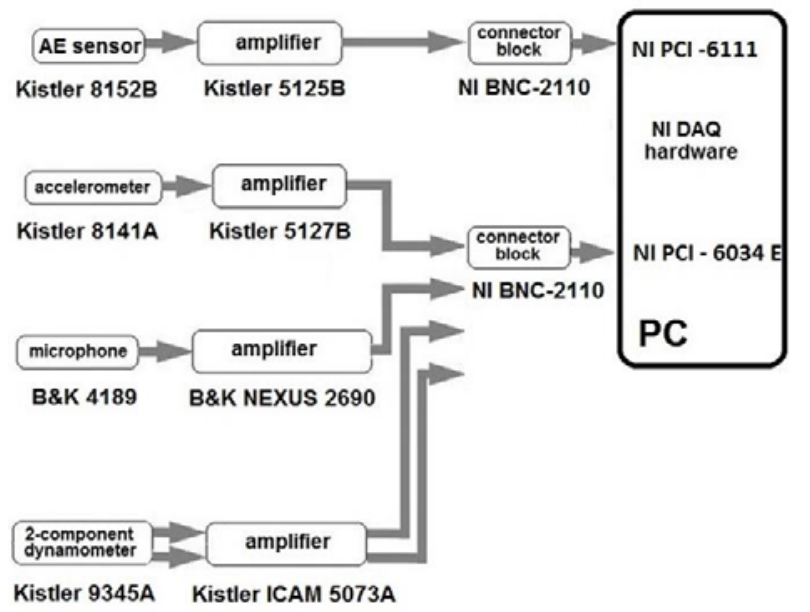

Fig. 1. The general scheme of devices used in the proposed online diagnostic system of the drill in laminated chipboard drilling process.

The quality of drilling results was controlled manually by human expert, who took into account the smoothness of the circumference and tolerance parameters specified in furniture manufacturing. The examples of acceptable and unacceptable holes drilled in a laminated chipboard are shown in Fig. 2.

The database for numerical experiments was prepared using 6 drills. All of them were used repeatedly to generate the proper size of learning data. In the first phase the new drills were used in laminated chipboard drilling process.

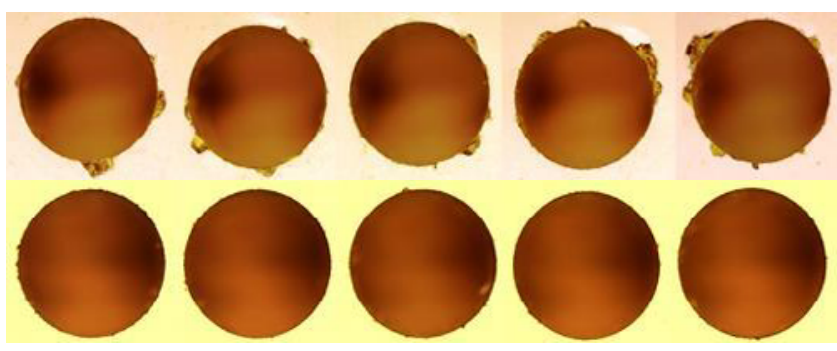

Fig. 2. The typical examples of unacceptable (upper row) and acceptable (lower row) holes drilled in a laminated chipboard.

The results were assessed by the human expert, responsible for the product quality. These samples represented observations belonging to class one. In the next phases the drills were successively blunted few times in a controlled way under the microscope Mitutoyo TM-505. The drills have been blunted, every time exacerbating the degree of drill blunting. After each blunting the drilling process was repeated 5 times and the signals of five sensors were registered, delivering the samples representing class 2 . In this way the class 2 was represented by the data corresponding to different degree of blunting. The registered time samples were arranged in a vector forms and the size of all vectors was the same and equal 60000.

The performed experimental trials have created the database, which was used in further numerical 
experiments. Totally 102 measurements of five sensor signals referring to class one have been registered. The database of blunted drill states contained 145 observations belonging to second class. This way the total number of observations, each representing five time series, was equal 247.

\section{Generation of diagnostic features}

The diagnostic features used in classification have been created on the basis of 5 measured signals: feed force $(\mathrm{F})$, cutting torque $(\mathrm{M})$, noise $(\mathrm{C})$, vibration $(\mathrm{V})$ and acoustic emission (A). Because of different sampling frequencies: $50 \mathrm{kHz}$ for $\mathrm{F}, \mathrm{M}, \mathrm{C}, \mathrm{V}$ and $2 \mathrm{MHz}$ for $\mathrm{A}$. The A signal was re-interpolated into common $50 \mathrm{kHz}$.

An examples of typical force signals representing the sharp and blunt states of the drill are presented in Fig. 3.

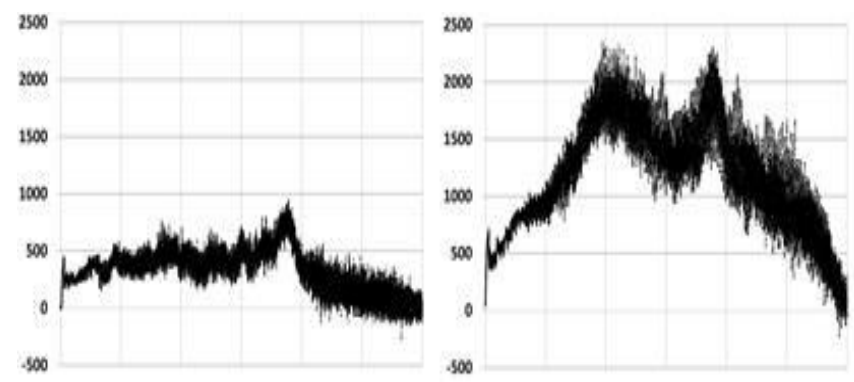

Fig. 3. The signals of the force registered for sharp (left) and blunt (right) states of the drill.

All five types of signals have been organized in the form of multidimensional time series $\mathbf{Y}$

$$
\mathbf{Y}=\left[\begin{array}{lllll}
\mathbf{F} & \mathbf{M} & \mathbf{C} & \mathbf{V} & \mathbf{A}
\end{array}\right]=\left[\begin{array}{lllll}
F_{1} & M_{1} & C_{1} & V_{1} & A_{1} \\
F_{2} & M_{2} & C_{2} & V_{2} & A_{2} \\
\mathrm{M} & \mathrm{M} & \mathrm{M} & \mathrm{M} & \mathrm{M} \\
F_{N} & M_{N} & C_{N} & V_{N} & A_{N}
\end{array}\right] \text { (1) }
$$

where $\mathrm{N}$ represents the size of the time series (60000 in experiments). The vector auto regression model (VAR) was applied in the generation of the diagnostic features [14]. VAR is a natural extension of the univariate autoregressive model to dynamic multivariate time series and forms one of the most successful, flexible, and easy to use model for the analysis of multivariate time series. Let

$$
\mathbf{y}_{t}=\left[\begin{array}{lllll}
F & M & C & V & A
\end{array}\right]^{T}
$$

denotes an $(5 \times 1)$ vector of time series variables. The basic p-lag vector autoregressive $\operatorname{VAR}(p)$ model has the form

$$
\mathbf{y}_{t}=\mathbf{A}_{1} \mathbf{y}_{t-1}+\mathbf{A}_{2} \mathbf{y}_{t-2}+\ldots+\mathbf{A}_{p} \mathbf{y}_{t-p}+\boldsymbol{\varepsilon}
$$

where $\mathbf{A}_{i}$ are $(5 \times 5)$ coefficient matrices and $\boldsymbol{\varepsilon}$ represents an $(5 \times 1)$ unobservable zero mean white noise vector process. The first order VAR(1) model of auto regression was used in our solution. This rank was chosen arbitrarily to reduce the risk of overfitting the model. In this case the auto regression process is defined as follows

$\left[\begin{array}{l}F_{t} \\ M_{t} \\ C_{t} \\ V_{t} \\ A_{t}\end{array}\right]=\left[\begin{array}{ccccc}a_{11} & a_{12} & a_{13} & a_{14} & a_{15} \\ a_{21} & a_{22} & a_{23} & a_{24} & a_{25} \\ \mathrm{M} & \mathrm{M} & \mathrm{M} & \mathrm{M} & \mathrm{M} \\ a_{51} & a_{52} & a_{53} & a_{54} & a_{55}\end{array}\right]\left[\begin{array}{l}F_{t-1} \\ M_{t-1} \\ C_{t-1} \\ V_{t-1} \\ A_{t-1}\end{array}\right]+\left[\begin{array}{c}\varepsilon_{F t} \\ \varepsilon_{M t} \\ \varepsilon_{C t} \\ \varepsilon_{V t} \\ \varepsilon_{A t}\end{array}\right](4)$

or in a compact matrix form

$$
\mathbf{y}_{t}=\mathbf{A}_{1} \mathbf{y}_{t-1}+\boldsymbol{\varepsilon}_{t}
$$

The auto regression coefficients forming matrix $\mathbf{A}_{1}$ have been estimated using maximum likelihood method $[14,15]$. In the case of data used in this work the matrices

\begin{tabular}{|c|c|c|c|c|c|}
\hline \multirow{5}{*}{$\mathbf{A}=$} & 0.9873 & 0.0015 & -0.0946 & -0.0041 & -0.0015 \\
\hline & 0.0016 & 0.9938 & -0.0253 & -0.0003 & -0.0046 \\
\hline & -0.0006 & 0.0018 & -0.0041 & 0.0052 & -0.0058 \\
\hline & 0.0006 & 0.0011 & -0.0029 & 0.8805 & 0.0042 \\
\hline & -0.0001 & -0.0010 & 0.0041 & 0.0005 & 0.2000 \\
\hline \multicolumn{6}{|c|}{ Blunt drill } \\
\hline \multirow{5}{*}{$\mathbf{A}=$} & 0.9894 & 0.0012 & -0.1284 & -0.0089 & -0.0041 \\
\hline & 0.0030 & 0.9983 & 0.0410 & 0.0027 & 0.0042 \\
\hline & -0.0027 & 0.0012 & 0.4894 & 0.0050 & -0.0002 \\
\hline & 0.0079 & -0.0001 & 0.0030 & 0.8943 & -0.0066 \\
\hline & 0.0000 & 0.0000 & -0.0010 & -0.0011 & 0.4947 \\
\hline
\end{tabular}
A obtained for the sharp and worn out drills are as following

- Sharp drills

The highest differences between the elements of $\mathbf{A}$ matrix for both types of drills are observed in the third (noise) and fifth (acoustic emission) columns. It means that these two signals will represent the highest ability of class discrimination.

The elements of auto regression matrix will form the basis for computation of the diagnostic features of the process. Three groups of features have been considered.

- Features equal to the autoregressive coefficients $a_{i j}$ of the matrix $\mathbf{A}_{1}$.

- Features based on the statistical description of each signal.

- Features based on the statistical description of the residual signals from VAR(1) auto regression.

The following statistical parameters have been applied in definition of the last two groups of features:
1. Mean
2. Median
3. Standard deviation (std)
4. Interquartile ranges (IQR) the ratio of IQR/median std/mean
7. Skewness
8. Cumulant of the third order
9. Cumulant of the fourth order

5. Interquartile coefficient of variation defined as

6. Coefficient of variation defined as the ratio

10. Cumulant of the fifth order.

On the basis of these descriptors 125 potential diagnostic features have been defined. Not all of them represented good class discrimination ability. After introductory 
experiments we have found that the most class distinctive are the features defined on the basis of residual signals from VAR(1) auto regression. They are defined for all 5 signals. Hence their total number is 50 . Only this set of features was investigated in the next stages of selection.

\section{Selection of diagnostic features}

The feature selection is the process of selecting a subset of relevant features, called also variables or predictors, for use in construction of the classification model. The most important reason of this step is to enhance the generalization ability of the classifier system by reducing the possible overfitting.

The central premise when using a feature selection technique is that the data contain features which are either redundant or irrelevant. Therefore, such features can be removed without significant loss of information $[16,17]$.

In our solution we have applied the sequentially repeated step wise approach. Let us assume $n$ potential features $x_{\mathrm{i}}(i=1,2, \ldots, n)$ subject to selection. The algorithm starts from some randomly chosen set of $m$ features $(m \leq n)$. Let us assume some measure of the quality $Q$ of the model. It might be the accuracy, sensitivity, area under ROC curve or their weighted combinations, reflecting the performance of the classifier at application of this subset as the input attributes. The higher is the value of this measure the better set of diagnostic features. By sequential adding and removing the features to the tested subsets of features we find the subset of the highest quality measure.

In this particular application the quality of the feature set was defined as the average of accuracy (ACC) of class recognition and the area under ROC curve (AUC) $[16,17]$, so $\mathrm{Q}=0.5(\mathrm{ACC}+\mathrm{AUC})$. The values of $\mathrm{ACC}$ and AUC are estimated as the average of the results of 10fold cross validation of the data in the classification mode. The classification system applied in this and other phases of signal processing was built on the basis of support vector machine of the Gaussian kernel.

\section{SVM classifier}

The diagnostic features selected in the previous stage are applied as the set of input attributes to the SVM classifier [18]. This solution of the classification system was chosen due to its very high efficiency in pattern recognition problems.

The support vector machine, developed by Vapnik, is a classification and regression system, characterized by very good generalization properties. It works in a space formed by the non-linear mapping of the original input vectors $\mathbf{y}$ into a feature space using the kernel function $\mathrm{K}\left(\mathbf{x}, \mathbf{x}_{i}\right)$. The learning problem of SVM is defined as the optimization task of separating the learning vectors $\mathbf{x}_{i}$ into two binary classes of the destination values either $d=1$ (one class) or $d=-1$ (the opposite class), with the maximal separation margin. The SVM training algorithm creates a model that assigns new data vectors into one class or the other on the basis of their location towards the separating hyperplane.
The important advantage of SVM is the special formulation of the learning problem, which leads to the quadratic programming with linear constraints. This type of optimization is very well developed and easy to solve. The separation margin introduced in the formulation of the minimized cost function provides some immunity of this classifier to the noise. Therefore, this approach to the classification problems has a reputation of very good generalization ability [18].

The effectiveness of SVM depends on the selection of the type of kernel, the kernel's parameters and the regularization parameter $C$. A common choice is a linear or Gaussian kernel, treated as the most universal and robust. Both are controlled by a single parameter $\gamma$. The optimal values of $\mathrm{C}$ and $\gamma$ have been selected by a grid search of their predefined values.

\section{Results of numerical experiments}

\subsection{Experimental setup}

The data set taking part in experiments contained 102 observations regarding the sharp state of drills and 145 observations representing different degrees of blunting (worn out state) [19]. They resulted in the total number of $n=247$ patterns, each representing 5 types of signals. They have been mixed and taken part in learning and testing the classification systems by applying leave-oneout mode of operation. Three variants of the investigations have been performed on the available measured data set.

- Selection of feature applying the whole data set and performing the classification in leave one out mode on the same set of patterns. One common set of selected features was used in learning all classification systems.

- Selection of the features combined with learning the SVM classifier on $\mathrm{n}-1$ patterns. The remaining nth pattern is left for testing the adapted system. The experiments are repeated $n$ times, each time with a new testing sample. The succeeding trials were performed at the newly selected sets of features.

- The data split into two separate parts. $40 \%$ of them are used in feature selection and the remaining $60 \%$ used only in learning and testing the classification property of the system in leave one out mode. One common set of features chosen in the selection phase is used in all these learning procedures of the SVM.

\subsection{Results of experiments}

The results of experiments will correspond to these three selection methods. In the first variant of investigation the selection process was done on the whole set of observations. On the basis of these results the selected features were fixed and applied as the input attributes in leave one out mode of classification. The samples used in classification were the same as in the feature selection. Two features have occurred to be the best: the interquartile ranges IQR of the noise $\mathrm{C}$ (notation $\mathrm{C} 4$ ) and the ratio $\mathrm{IQR} /$ median of the acoustic emission (notation A5). The other features have been assessed by the selection algorithm as not important in this variant of 
investigations. The application of the linear SVM of the regularization constant equal 100 has allowed to obtain the average accuracy equal $\mathrm{ACC}=0.976$.

The second variant of investigations is more demanding, since the selection of features and the process of learning was repeated $n$ times ( $n-$ the number of observations). Each time the learning procedure was performed on $n-1$ cases, leaving the $n$th one for testing. In all runs of experiments the set of selected features was under change. Fig. 4 presents the relative histogram of how often the particular features have been chosen by the selection algorithm. The notations of the features correspond to the name of signal $(\mathrm{V}, \mathrm{C}, \mathrm{F}, \mathrm{M}, \mathrm{A})$ and the succeeding number of the statistics (from 1 to 10) used in generation of the particular feature. As it is seen the statistics of the features $\mathrm{C} 4$ and A5 have been the most often chosen by the selection algorithm in all runs of the leave one out experiments. The average accuracy rate obtained in the experiments in this variant was similar to the previous case and equal $\mathrm{ACC}=0.967$.

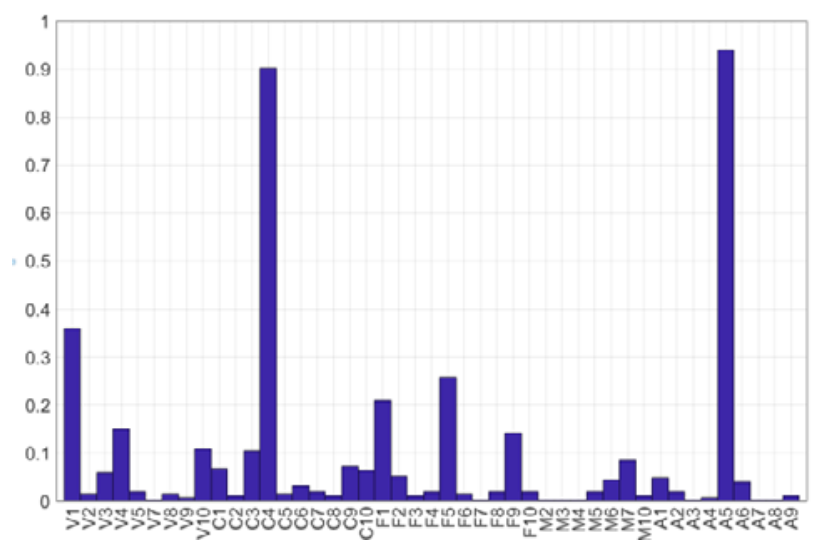

Fig. 4. The percentage of times the features have been selected in the second variant of the experiments

The last variant of experiments was the most demanding. The selection stage was separated from the classification and testing. The data set was split into $40 \%$ used only in selection of the features and the remaining $60 \%$ of the data used only in the classification and testing. The classification of the data has been also done in leave one out mode of operation. This variant of investigation is the most similar to the future application of the system in industry.

Fig. 5 shows the percentage of times the particular features have been chosen by the selection algorithm in 2000 repetitions of the experiments. It is seen that larger population of features has been selected as the input attributes in this variant of experiments.

The accuracy of class recognition varied from run to run. Fig. 6 shows the histogram of this accuracy values in 2000 repetitive runs of algorithm. The average accuracy of class recognition obtained in these experiments was equal $\mathrm{ACC}=0.950$.

Another quality measure of the classification system is the Receiver Operating Characteristics (ROC). Especially important is the area under curve (AUC). Good classifier should have this value close to one. On the other side the random classifier is characterized by this value equal 0.5 . In our solution the AUC was equal 0.947 , which is very close to be ideal.

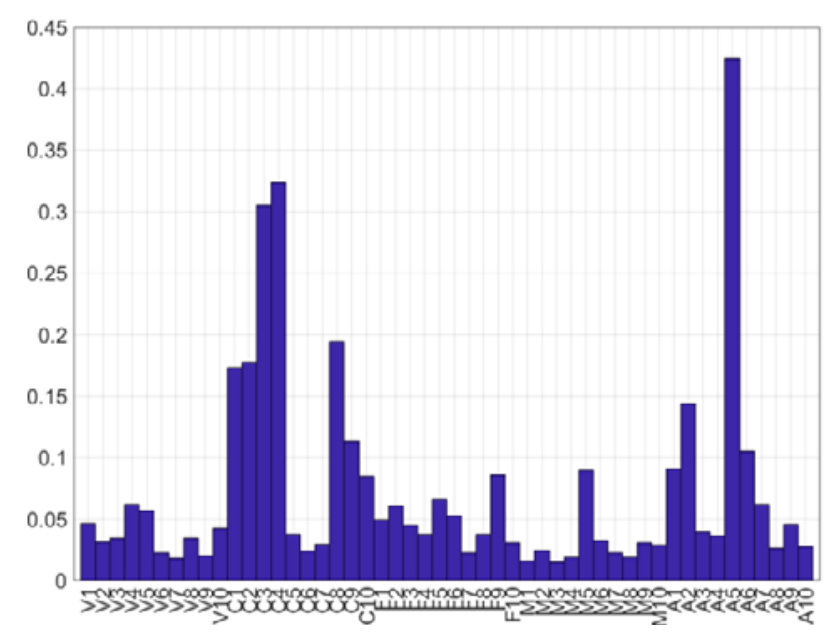

Fig. 5. The percentage of times the features have been selected in the third variant of the experiments

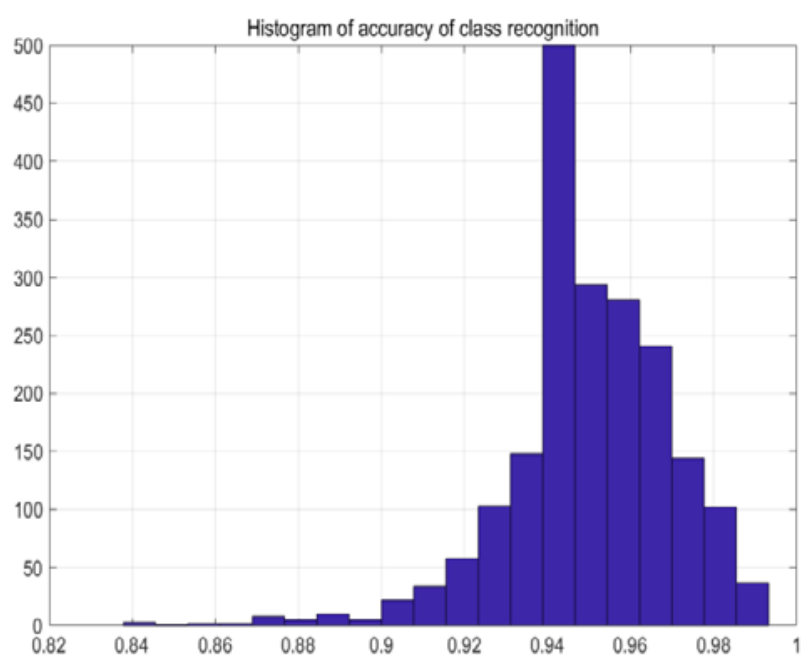

Fig. 6. The histogram of accuracy of class recognition in 2000 runs of the third variant of experiments

\section{Conclusions}

The paper has presented an automatic system for recognition of the condition of drill in a standard laminated chipboard drilling process. Two states of drill have been recognized: the sharp one, allowing the process of drilling to continue and blunt one, which should lead to stop the production. The recognition process is done on-line without interrupting the drilling process.

The presented solution shows that the satisfactory results of recognition of drill condition are possible online by proper processing the sensor signals registering the feed force, cutting torque, noise, vibration and acoustic emission. On the basis of these signals the diagnostic features have been defined using the multivariable auto regression model VAR(1). The selection process following the generation of these features was responsible for selecting the most significant ones, which were used as the input attributes to the classification system. 
The support vector machine classifier was used for the class recognition. Its choice was dictated by very good generalization ability of this solution. The results of its application are acceptable in practice. The relative testing error of recognition in leave-one-out mode of operation was changing from $3 \%$ to $5 \%$, depending on the organizational variant of the investigations.

\section{References}

[1] C. Scheffer, H. Kratz, P.S. Heyns, F. Klocke, Development of a tool wear-monitoring system for hard turning, International Journal of Machine Tools \& Manufacture, 43, 973-985 (2003).

[2] D. E. Dimla, P. M. Lister, On-line metal cutting tool condition monitoring. I: force, vibration analyses, International Journal of Machine Tools \& Manufacture, 40, 739-768 (2000).

[3] K. Jemielniak, T. Urbański, J. Kossakowska J., S. Bombiński, Tool condition monitoring based on numerous signal features, Int J. Adv. Manuf. Technol., 59, 73-81 (2012).

[4] N. H. Abu-Zahra, G. Yu, Gradual wear monitoring of turning inserts using wavelet analysis of ultrasound waves, International Journal of Machine Tools \& Manufacture, 43, 33-343 (2003).

[5] R. Lemaster, L. Lu, S. Jackson, The use of process monitoring techniques on a CNC wood router. Part 1. Sensor selection, Forest Products Journal, 50, 7/8, 31-64 (2000).

[6] J. Wilkowski, J. Górski, Vibro-acoustic signals as a source of information about tool wear during laminated chipboard milling, Wood Research, 56, 1, 57-66 (2011).

[7] R. G. Silva, K. J. Baker, S. J. Wilcox, The adaptability of a tool wear monitoring system under changing cutting conditions, Mechanical Systems, Signal Processing, 14, 2, 287-298 (2000).

[8] R. J. Kuo, Multi-sensor integration for on-line tool wear estimation through artificial neural networks, fuzzy neural network, Engineering Applications of Artificial Intelligence, 13, 249-261 (2000).

[9] A. Noori-Khajavi, R. Komandur, Frequency, time domain analyses of sensor signals in drilling-Part I, International Journal of Machine Tools, Manufacture, 35, 6, 775-793 (1995).

[10] J. H. Zhou, C. K. Pang, Z. W. Zhong, F. L. Lewis, Tool wear monitoring using acoustic emissions by dominant-feature identification, IEEE Transactions on Instrumentation, Measurement, 60, 2, 547-559 (2011).

[11] S. S. Panda, A. K. Singh, D. Chakraborty, S. K. Pal, Drill wear monitoring using back propagation neural network, Journal of Materials Processing Technology, 172, 283-290 (2006).

[12] K. Patra, S. K. Pal, K. Bhattacharyya, Artificial neural network based prediction of drill flank wear from motor current signals, Applied Soft Computing, 7, 929-935 (2007).

[13] P. Lezanski, An intelligent system for grinding wheel condition monitoring, Journal of Materials Processing Technology, 109, 258-263 (2001).

[14] H. Lutkepohl, Introduction to Multiple Time Series Analysis, Berlin Springer-Verlag (1991).

[15] Matlab user manual, Natick: MathWorks, (2014).

[16] P.N. Tan, M. Steinbach, V. Kumar, Introduction to data mining, Boston: Pearson Education Inc. (2006).

[17] J. Kurek, S. Osowski, Support vector machine for fault diagnosis of the broken rotor bars of squirrelcage induction motor, Neural Computing and Applications, 19, 557-564 (2010).

[18] V. Kecman, Learning, Soft Computing: Support Vector Machines, Neural Networks,, Fuzzy Logic Models, Cambridge, MA: MIT Press (2001).

[19] M. Kruk, A. Jegorowa, J. Kurek, S. Osowski, J. Górski, Automatic recognition of drill condition on the basis of images of drilled holes, in Proc. Conference "Computational Problems of Electrical Engineering”, Sandomierz, 2016, pp. 1-4 